\title{
Contrast-Enhanced CT Colonography Features of Rectal Carcinoid Tumors
}

\author{
Daisuke Tsurumaru, Satoshi Kawanami, Yusuke Nishimuta, Mitsutoshi Miyasaka, \\ Yoshiki Asayama, Hiroshi Honda \\ Department of Clinical Radiology, Graduate School of Medical Sciences, Kyushu University, Fukuoka, Japan \\ Email: tsuru-d@radiol.med.kyushu-u.ac.jp
}

Received 5 April 2014; revised 5 May 2014; accepted 12 May 2014

Copyright @ 2014 by authors and Scientific Research Publishing Inc.

This work is licensed under the Creative Commons Attribution International License (CC BY). http://creativecommons.org/licenses/by/4.0/

(c) (i) Open Access

\section{Abstract}

Purpose: The purpose of this study was to retrospectively assess the detectability and enhancement pattern of rectal carcinoid tumors on contrast-enhanced computed tomography colonography (CE-CTC). Methods: The study sample consisted of 12 lesions in nine patients of rectal carcinoid tumors. To evaluate the detectability, two radiologists reviewed axial and 3D images including air enema (AE), virtual endoscopy (VE), and virtual colon dissection (VCD). To determine the contrast enhancement pattern of the tumors, the CT attenuation value of each lesion was measured in three phases. Results: Four of the 12 lesions (33\%) were detected on axial images in patients in both the prone and supine positions, the sizes of which were $6 \mathrm{~mm}$ or larger. Seven lesions $(58 \%)$, including the four lesions mentioned above, were detected on 3D images including $\mathrm{AE}$ and $\mathrm{VE}$ in patients in the prone position, the sizes of which were $5 \mathrm{~mm}$ or larger. The ranges of CT attenuation values of the lesions at $40 \mathrm{~s}, 70 \mathrm{~s}$ and $240 \mathrm{~s}$ were $112-147,116-140$ and 82 - 97 HU, respectively. Conclusion: Rectal carcinoid tumors are challenging to detect on CE-CTC. They demonstrated enhancement at the early phase and washout at the delayed phase on CE-CTC.

\section{Keywords}

Carcinoid Tumors, Rectum, Contrast Enhancement, CT Colonography

\section{Introduction}

Carcinoid tumors belong to the group of well-differentiated neuroendocrine tumors of neuroendocrine neoplasms in the World Health Organization (WHO) classification [1]. Rectal carcinoid tumors are uncommon, representing $1.1 \%$ to $1.3 \%$ of all rectal tumors [2] [3]. They can show a broad range of clinical behavior, from benign and asymptomatic to disseminated and metastatic. Clinical behavior is a reflection of tumor size, with me- 
tastatic potential generally being proportional to size [4]-[6]. Rectal carcinoid tumors are usually found incidentally by rectal examination or endoscopy. Tumors smaller than $10 \mathrm{~mm}$ in diameter rarely metastasize, and thus have usually been managed with local excision including endoscopic treatment [7]-[9].

In preoperative workups, computed tomography (CT) is more useful in the demonstration of mesenteric extension and distant metastases. On endoscopy and CT, rectal carcinoid tumors appear as small solitary nodules, multiple masses, or a large polypoid ulcerating mass [10]. Although optical colonoscopy may help accurately examine mucosal lesions, the ability to fully characterize a submucosal abnormality on the sole basis of a luminal examination is somewhat limited. In addition, the diagnostic yield of endoscopic biopsy for such lesions is relatively low [11].

CT colonography (CTC) has become a useful diagnostic tool in colorectal cancer staging and screening, enabling structural evaluations of the colon and other abdominal organs through detailed one-dimensional and three-dimensional (3D) images that can accurately identify colonic and extracolonic pathologies. However, CTC features including the contrast enhancement pattern of rectal carcinoid tumors have been unclear. The purpose of this study was to retrospectively assess the detectability and enhancement pattern on contrast-enhanced CTC (CE-CTC) of rectal carcinoid tumors.

\section{Methods}

The institutional review board at our institution approved this retrospective study and waived the requirement for informed patient consent.

\subsection{Study Patients}

The study sample consisted of 12 lesions in nine consecutively registered patients (six men, three women; median age, 54 years) who were pathologically confirmed as having carcinoid tumor of the rectum between January 2007 and August 2013. All patients were endoscopically suspected to have rectal carcinoid tumors and were referred to our institution for further examinations or treatment. As the preoperative workup, all patients were assessed using optical colonoscopy, endosonography, and CE-CTC. All of the tumors were located in the submucosa of the middle to lower rectum, $9 \mathrm{~cm}$ from the anal verge [12], with no lymph nodes or distant metastases. All patients were treated by an endoscopic procedure, such as endoscopic mucosal resection or submucosal dissection. The tumor sizes ranged from 2.5 to $12.0 \mathrm{~mm}$ (median, $5 \mathrm{~mm}$ ) in maximum diameter (Table 1).

\subsection{Technique of Colonoscopy and CT Colonography}

Colonoscopy was immediately followed by same-day CE-CTC using the same bowel preparation. The prepara-

\begin{tabular}{lc} 
Table 1. Patients characteristics. \\
\hline Age (y), median & 54 (range $36-71)$ \\
Sex (M:F) & $6: 3$ \\
Location* & \\
$\quad$ Middle rectum & 4 \\
$\quad$ Lower rectum & 8 \\
Tumor size (mm), median & \\
Treatment & 10 \\
$\quad$ EMR & $2.5-12)$ \\
$\quad$ ESD & \\
Depth & 12 \\
$\quad$ Submucosa
\end{tabular}

EMR, endoscopic mucosal resection; ESD, endoscopic submucosal dissection. ${ }^{*}$ Middle rectum $=6-9 \mathrm{~cm}$ from anal verge; Lower rectum $=3-6 \mathrm{~cm}$ from anal verge. 
tions for the study examinations included the patient's oral ingestion of a $2000-\mathrm{mL}$ volume of isotonic polyethylene glycol electrolyte solution (Niflec ${ }^{\mathbb{B}}$; Ajinomoto Pharma. Co., Tokyo) in the morning of the day on which the CTC was performed.

Total colonoscopy was performed to verify the rectal lesion and to screen the entire large intestine. After the rectal lesion was identified, a biopsy was performed, and seven of the 12 lesions were pathologically diagnosed as carcinoid tumors preoperatively. The other five lesions were clinically diagnosed as carcinoid tumors of the rectum by endoscopic and endosonographic findings. Before the CT scanning, bowel distension was obtained by room air gently insufflated in the colon via a transrectal catheter, and $20 \mathrm{mg}$ of scopolamine (Buscopan; Boehringer Ingelheim, Tokyo) was administered intravenously to allow optimal colonic distension, to minimize peristalsis, and to alleviate spasms.

All CT examinations were performed using a 64-detector-row CT scanner (Aquilion, Toshiba Medical Systems, Tokyo) according to the following protocol: $120 \mathrm{kV}, 200-300 \mathrm{~mA}$ with automatic exposure control, 64 rows $\times 1 \mathrm{~mm}$ collimation, helical pitch 53.0. CT images were obtained $40 \mathrm{~s}, 70 \mathrm{~s}$ and $240 \mathrm{~s}$ after the injection of $120 \mathrm{~mL}$ nonionic contrast material (Iopamiron370; Bayer Health Care, Osaka, Japan) at a rate of $3 \mathrm{~mL} / \mathrm{s}$ with the patient in the supine position ( $40 \mathrm{~s}$ and $70 \mathrm{~s})$ and in the prone position $(240 \mathrm{~s})$. The multi-detector row computed tomography (MDCT) data sets were loaded onto a 3D workstation, Synapse Vincent (Fujifilm Medical, Tokyo). After all image data sets were transferred onto the workstation, they were converted into 3D imagesair enema (AE), virtual endoscopy (VE), and virtual colon dissection (VCD) [13]—using the workstation's onboard software.

\subsection{Image Analysis}

The image analysis was performed by two gastrointestinal radiologists, with seven and 12 years of experience, respectively. Before the image interpretation, information that one or more carcinoid tumors were present in the rectum was given, but the readers were blinded to the location and size of the lesions. First, the readers reviewed the axial images of all three phases on a 21-inch PACS monitor (Rapideye Station; Toshiba Medical Systems). Thereafter, they reviewed the AE, VE and VCD images of all three phases on a 20 inch workstation monitor at over 2 weeks intervals. To evaluate the detectability, the readers determined the presence or absence of rectal carcinoid tumors. A lesion was determined to be present when a protruded, submucosal tumor-like, nodule or mass, with or without contrast enhancement on axial images, was visible in the rectum. Differences in assessments were resolved by consensus. The localization of the lesion(s) was precisely determined by the optical endoscopic findings as a standard of reference. To determine the contrast enhancement pattern of the rectal carcinoid tumors, the CT attenuation value of each lesion was measured by the largest possible circular region-ofinterest in all phases.

\section{Results}

The results are shown in Table 2. There was no discordance between the readers in lesion detection for all images. Four of the 12 lesions (33\%) were detected on axial images in patients in both the prone and supine positions, the sizes of which were $6 \mathrm{~mm}$ or larger. Seven lesions (58\%), including the four lesions mentioned above, were detected on $3 \mathrm{D}$ images including $\mathrm{AE}$ and $\mathrm{VE}$ in patients in the prone position, the sizes of which were 5 $\mathrm{mm}$ or larger. Two lesions (case nos.1 and 9) the sizes of which were $5 \mathrm{~mm}$ in diameter were detected on 3D images but not detected on axial images only (Figure 1). In one case (no. 5) with a $5 \mathrm{~mm}$ diameter lesion, the readers detected the tumor on $\mathrm{AE}$ and $\mathrm{VE}$ taken in the prone position, but did not detect it on VCD with either the supine or prone position. There were no false positive cases in this detectability test. All four lesions detected on the axial images (6 mm or larger) demonstrated enhancement at the early phase ( $40 \mathrm{~s}$ and $70 \mathrm{~s})$, and washout at the delayed phase (240 s) (Figure 2). The ranges of CT attenuation values at $40 \mathrm{~s}, 70 \mathrm{~s}$ and $240 \mathrm{~s}$ were 112 - 147, 116 - 140 and 82 - $97 \mathrm{HU}$, respectively (Figure 3).

\section{Discussion}

The present study revealed that four of 12 carcinoid tumors (33\%) with the size of $6 \mathrm{~mm}$ or larger were detected on axial images. Seven lesions (58\%) $5 \mathrm{~mm}$ or larger were detected with the use of 3D images. In the case of colorectal polyps, the sensitivity of CTC has been shown to be directly related to polyp size, with the sensitivity 
Table 2. Detectability of the rectal carcinoid tumors on CTC.

\begin{tabular}{ccccccc}
\hline \multirow{2}{*}{ Case no. } & Diameter $(\mathbf{m m})$ & Axial & \multicolumn{4}{c}{ 3D CT } \\
\cline { 3 - 7 } 1 & & Total & AE & VE & VCD \\
\hline 2 & 5.0 & No & Yes & Yes & Yes & Yes \\
& 12.0 & Yes & Yes & Yes & Yes & Yes \\
3 & 8.0 & Yes & Yes & Yes & Yes & Yes \\
& 4.5 & No & No & No & No & No \\
4 & 2.5 & No & No & No & No & No \\
5 & 8.0 & Yes & Yes & Yes & Yes & Yes \\
6 & 5.0 & No & Yes & Yes ${ }^{*}$ & Yes ${ }^{*}$ & No \\
7 & 6.0 & Yes & Yes & Yes & Yes & Yes \\
& 4.0 & No & No & No & No & No \\
8 & 5.0 & No & No & No & No & No \\
9 & 5.0 & No & No & No & No & No \\
& 5.0 & No & Yes & Yes & Yes & Yes \\
& & $4 / 12$ & $7 / 12$ & $7 / 12$ & $7 / 12$ & $6 / 12$ \\
\hline
\end{tabular}

AE, air enema; VE, virtual endoscopy; VCD, virtual colon dissection. ${ }^{*}$ Tumors not detected in supine position.

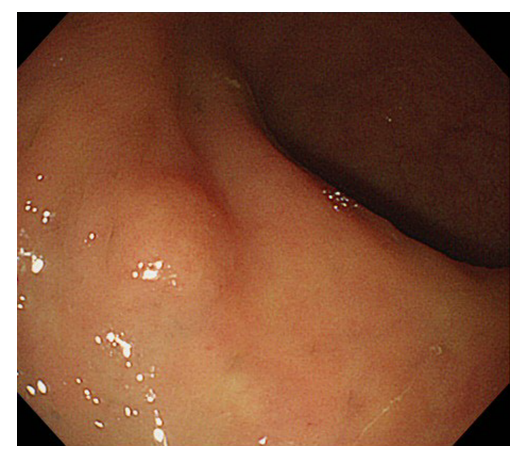

(a)

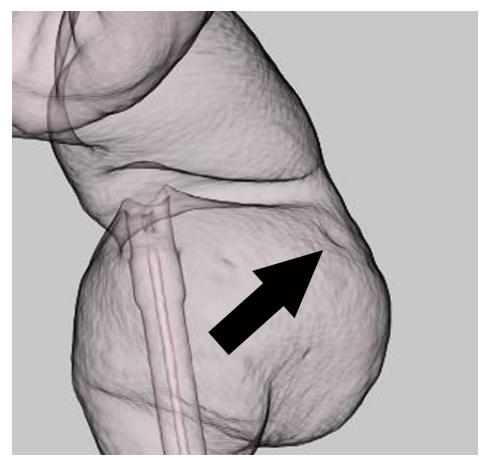

(d)

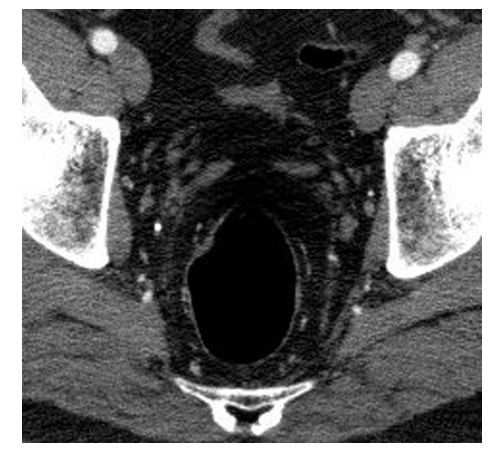

(b)

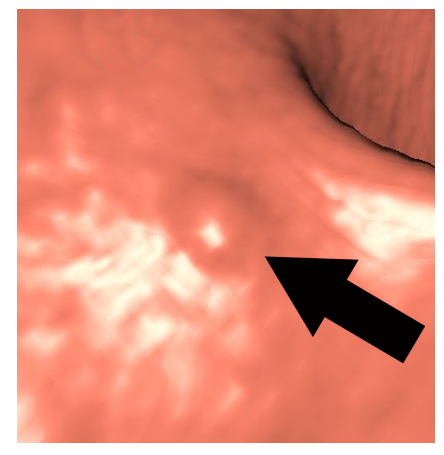

(c)

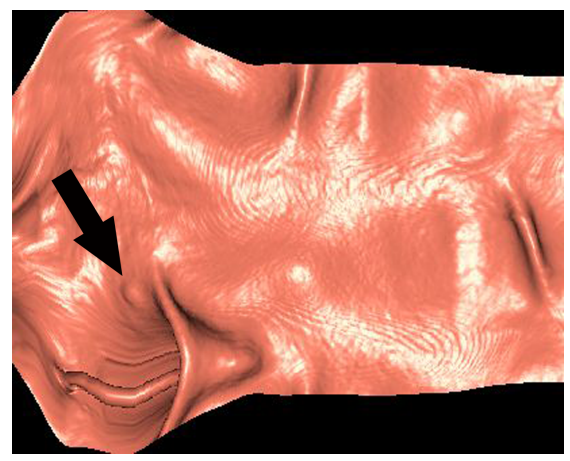

(e)

Figure 1. 37-year-old man with rectal carcinoid tumor with the size of 5mm in diameter (case no. 9). (a) Optical colonoscopy shows smooth-surfaced submucosal tumor located in the rectum. (b) The rectal carcinoid tumor is not evident on axial images. There seems to be focal wall thickening at the 10-o'clock position in the rectum, which is a partial visualization of normal fold. The carcinoid tumor of this case locates in the left wall of the rectum. (c) Virtual endoscopy shows smooth-surfaced tumor in the rectum (arrow). (d) Air enema shows smooth-surfaced tumor in the left wall of the rectum (arrow). (e) The tumor is also detectable on virtual colon dissection (arrow). 


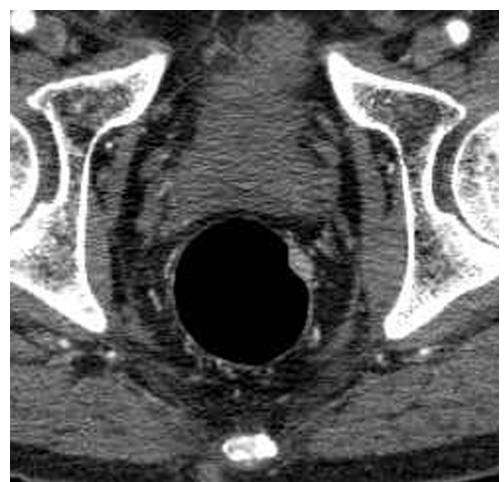

(a)

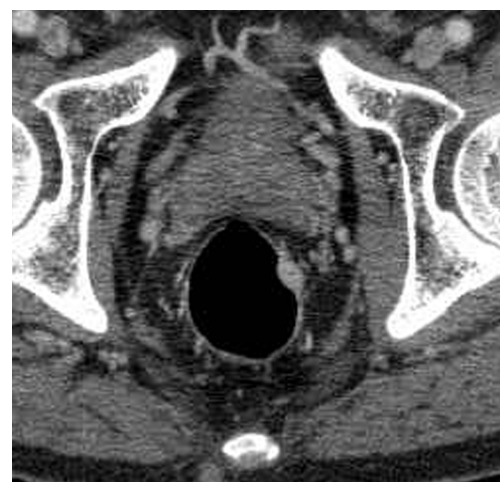

(b)

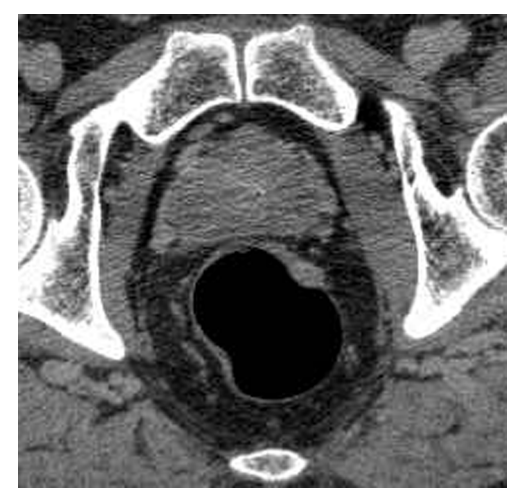

(c)

Figure 2. 39-year-old man with rectal carcinoid tumor (case no. 4). The tumor is well enhanced at the early phase (a) and the enhancement washout at the delayed phase (b) on CE-CTC.

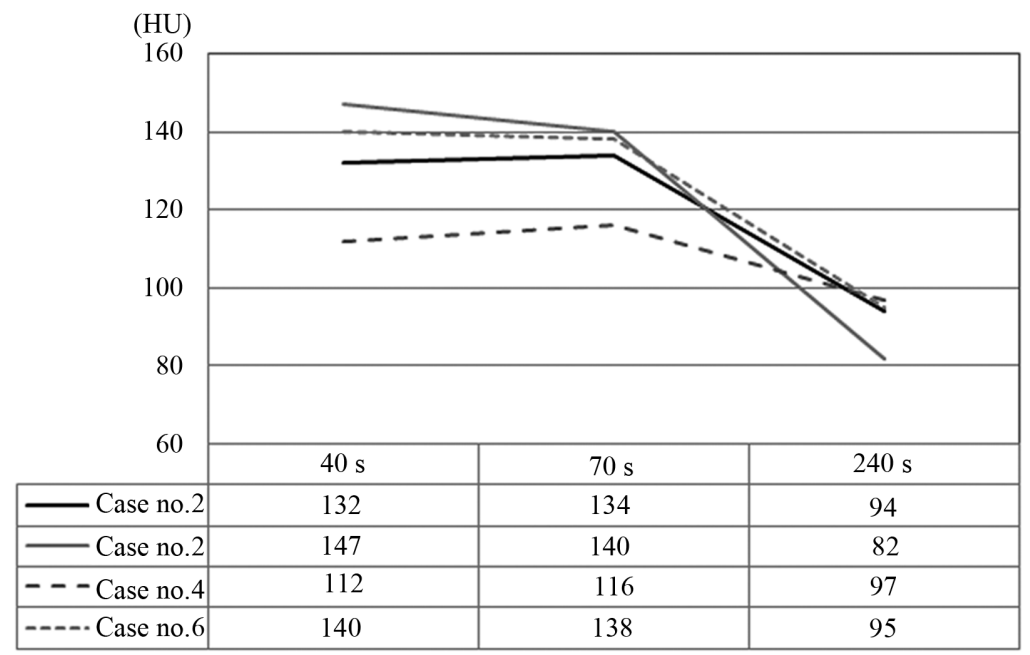

Figure 3. CT attenuation value of rectal carcinoid tumors detected on axial images ( $6 \mathrm{~mm}$ or larger). All of four lesions shows enhancement at the early phase (40 s and $70 \mathrm{~s})$, and washout at the delayed phase ( $240 \mathrm{~s})$.

of CTC for the detection of polyps $5 \mathrm{~mm}$ or smaller in the range of $10 \%-67 \%$ [14]. Carcinoid tumors may be less detectable than mucosal polyps on CTC because the carcinoid tumors manifest as submucosal tumors, which are smooth-surfaced and have ill-defined margins.

However, in the present patient series the smaller lesions which were not detectable on axial images could only be detected with the addition of 3D CT. In one patient (case no. 5) with a rectal carcinoid tumor $5 \mathrm{~mm}$ in diameter, the readers detected the tumor on AE and VE with the patient in the prone position, but they did not detect the tumor with the patient in the supine position. The main causes of missed or overcalled lesions by CTC are poorly distended segments, inadequate bowel preparation, and flat mucosal lesions. Collapsed colonic segments may be misinterpreted as annular neoplasms or may preclude the detection of flat lesions or significant polyps [15]. In particular, rectal distention is poorer when patients are in the supine position [16]. In the same case in our series (case no. 5), the tumor was not detected on VCD with the use of either the supine or prone position. Lee et al. mentioned that the image distortion on VCD makes polyps look unfamiliar to readers, thus causing unnecessary inconvenience in interpretation and necessitating additional training for readers to become accustomed to the unnatural appearances of polyps [17].

In the present study, carcinoid tumors revealed well-enhanced lesions in the early phase, and washout at the delayed phase after the administration of intravenous contrast agent. Karen et al. noted that carcinoid tumors of the gastrointestinal tract can be seen as dramatically enhancing submucosal lesions, because of their increased vascularity [18]. Carcinoid tumors of the stomach, duodenum and small intestine also show early-phase en- 
hancement patterns on contrast-enhanced CT [10] [11].

Colorectal adenomas and adenocarcinomas also demonstrate enhancement on CE-CTC. Oto et al. reported that the mean $\mathrm{CT}$ attenuation value of colorectal adenomas and adenocarcinomas $70 \mathrm{~s}$ after the administration of an intravenous contrast agent were $78.9 \pm 4.4$ and $90.7 \pm 2.1 \mathrm{HU}$, respectively [19]. In the present study, all carcinoid tumors for which attenuation was measured on CE-CTC had values of more than $112 \mathrm{HU}$ at the early phase, $40 \mathrm{~s}$ or $70 \mathrm{~s}$ after the administration of the contrast agent. Although the study sample and/or examination methods differ among studies, the CT values or enhancement pattern on CE-CTC may be key features in differrentiating carcinoid tumors from colorectal adenomas or adenocarcinomas.

Optical colonoscopy contributes to the accurate differentiation of mucosal lesions from lesions of submucosal origin, but the ability to fully characterize a submucosal abnormality on the sole basis of a luminal examination is somewhat limited because the diagnostic yield of endoscopic biopsy for such lesions is relatively low [11]. In five of 12 lesions in the present patient series, the diagnosis of the lesion as a carcinoid tumor could not be accomplished preoperatively with the biopsy specimen.

CE-CTC is a useful noninvasive diagnostic tool for carcinoid tumors of the colorectal region. In the present study, three patients had multiple lesions. The second carcinoid tumors were incidentally discovered by optical colonoscopy in patients known to have a carcinoid tumor in the rectum. CE-CTC may also be useful in screening for concurrent second lesions of rectal carcinoid tumors, as is true of optical colonoscopy.

The present study had several limitations. First, this retrospective study included a small number of patients. Second, we did not have the data of precontrast CTC. We applied our institution's standard CT protocol for colorectal cancers, which included CE-CTC only, to carcinoid tumors. Precontrast CTC is essential to accurately assess the enhancement pattern of carcinoid tumors. Third, in clinical settings, the significance of performing CTC for rectal carcinoid tumors is controversial. Endosonography is a commonly used technique in the diagnosis of rectal carcinoid tumors with high accuracy [20] [21]. The clinical information of rectal carcinoid tumors obtained by CE-CTC might not exceed that obtained by endosonography.

\section{Conclusion}

In conclusion, rectal carcinoid tumors are challenging to detect on CE-CTC. However, the detection rate increased with the addition of 3DCT. Rectal carcinoid tumors demonstrated enhancement at the early phase and washout at the delayed phase on CE-CTC. These features may play an important role in differentiating carcinoid tumors from other rectal tumors.

\section{References}

[1] Bosman, F.T. (2010) WHO Classification of Tumours of the Digestive System. World Health Organization Classification of Tumours, 4th Edition, International Agency for Research on Cancer, Lyon.

[2] Godwin, J.D., 2nd (1975) Carcinoid Tumors. An Analysis of 2837 Cases. Cancer, 36, 560-569. http://dx.doi.org/10.1002/1097-0142(197508)36:2<560::AID-CNCR2820360235>3.0.CO;2-4

[3] Teleky, B., Herbst, F., Langle, F., Neuhold, N. and Niederle, B. (1992) The Prognosis of Rectal Carcinoid Tumours. International Journal of Colorectal Disease, 7, 11-14. http://dx.doi.org/10.1007/BF01647653

[4] Modlin, I.M., Lye, K.D. and Kidd, M. (2003) A 5-Decade Analysis of 13,715 Carcinoid Tumors. Cancer, 97, $934-959$. http://dx.doi.org/10.1002/cncr.11105

[5] Fahy, B.N., Tang, L.H., Klimstra, D., Wong, W.D., Guillem, J.G., Paty, P.B., Temple, L.K., Shia, J. and Weiser, M.R. (2007) Carcinoid of the Rectum Risk Stratification (CaRRs): A Strategy for Preoperative Outcome Assessment. Annals of Surgical Oncology, 14, 1735-1743. http://dx.doi.org/10.1245/s10434-006-9311-6

[6] Koura, A.N., Giacco, G.G., Curley, S.A., Skibber, J.M., Feig, B.W. and Ellis, L.M. (1997) Carcinoid Tumors of the Rectum: Effect of Size, Histopathology, and Surgical Treatment on Metastasis Free Survival. Cancer, 79, $1294-1298$. http://dx.doi.org/10.1002/(SICI)1097-0142(19970401)79:7<1294::AID-CNCR4>3.0.CO;2-H

[7] Soga, J. (2005) Early-Stage Carcinoids of the Gastrointestinal Tract: An Analysis of 1914 Reported Cases. Cancer, 103, 1587-1595. http://dx.doi.org/10.1002/cncr.20939

[8] Mashimo, Y., Matsuda, T., Uraoka, T., Saito, Y., Sano, Y., Fu, K., Kozu, T., Ono, A., Fujii, T. and Saito, D. (2008) Endoscopic Submucosal Resection with a Ligation Device Is an Effective and Safe Treatment for Carcinoid Tumors in the Lower Rectum. Journal of Gastroenterology and Hepatology, 23, 218-221. http://dx.doi.org/10.1111/j.1440-1746.2008.05313.x 
[9] Park, H.W., Byeon, J.S., Park, Y.S., Yang, D.H., Yoon, S.M., Kim, K.J., Ye, B.D., Myung, S.J., Yang, S.K. and Kim, J.H. (2010) Endoscopic Submucosal Dissection for Treatment of Rectal Carcinoid Tumors. Gastrointestinal Endoscopy, 72, 143-149. http://dx.doi.org/10.1016/j.gie.2010.01.040

[10] Elsayes, K.M., Menias, C.O., Bowerson, M., Osman, O.M., Alkharouby, A.M. and Hillen, T.J. (2011) Imaging of Carcinoid Tumors: Spectrum of Findings with Pathologic and Clinical Correlation. Journal of Computer Assisted Tomography, 35, 72-80. http://dx.doi.org/10.1097/RCT.0b013e3181fd2044

[11] Pickhardt, P.J., Kim, D.H., Menias, C.O., Gopal, D.V., Arluk, G.M. and Heise, C.P. (2007) Evaluation of Submucosal Lesions of the Large Intestine: Part 2. Nonneoplastic Causes. Radiographics, 27, 1693-1703. http://dx.doi.org/10.1148/rg.276075028

[12] Salerno, G., Sinnatamby, C., Branagan, G., Daniels, I.R., Heald, R.J. and Moran, B.J. (2006) Defining the Rectum: Surgically, Radiologically and Anatomically. Colorectal Disease, 8, 5-9. http://dx.doi.org/10.1111/j.1463-1318.2006.01062.x

[13] Hoppe, H., Quattropani, C., Spreng, A., Mattich, J., Netzer, P. and Dinkel, H.P. (2004) Virtual Colon Dissection with CT Colonography Compared with Axial Interpretation and Conventional Colonoscopy: Preliminary Results. American Journal of Roentgenology, 182, 1151-1158. http://dx.doi.org/10.2214/ajr.182.5.1821151

[14] Macari, M., Bini, E.J., Jacobs, S.L., Lui, Y.W., Laks, S., Milano, A. and Babb, J. (2004) Significance of Missed Polyps at CT Colonography. American Journal of Roentgenology, 183, 127-134. http://dx.doi.org/10.2214/ajr.183.1.1830127

[15] Morrin, M.M., Farrell, R.J., Keogan, M.T., Kruskal, J.B., Yam, C.S. and Raptopoulos, V. (2002) CT Colonography: Colonic Distention Improved by Dual Positioning But Not Intravenous Glucagon. European Radiology, 12, 525-530. http://dx.doi.org/10.1007/s003300100954

[16] Taylor, S.A., Halligan, S., Goh, V., Morley, S., Bassett, P., Atkin, W. and Bartram, C.I. (2003) Optimizing Colonic Distention for Multi-Detector Row CT Colonography: Effect of Hyoscine Butylbromide and Rectal Balloon Catheter. Radiology, 229, 99-108. http://dx.doi.org/10.1148/radiol.2291021151

[17] Lee, S.S., Park, S.H., Kim, J.K., Kim, N., Lee, J., Park, B.J., Kim, Y.J., Lee, M.W., Kim, A.Y. and Ha, H.K. (2009) Panoramic Endoluminal Display with Minimal Image Distortion Using Circumferential Radial Ray-Casting for Primary Three-Dimensional Interpretation of CT Colonography. European Radiology, 19, 1951-1959. http://dx.doi.org/10.1007/s00330-009-1362-1

[18] Veyrie, N., Pierre-Kahn, N., Hugol, D., Servajean, S., Berger, N., Bethoux, J.P. and Bouillot, J.L. (2004) Carcinoid Tumor from the Small Bowel. Annales de Chirurgie, 129, 607-610. http://dx.doi.org/10.1016/j.anchir.2004.09.012

[19] Oto, A., Gelebek, V., Oguz, B.S., Sivri, B., Deger, A., Akhan, O. and Besim, A. (2003) CT Attenuation of Colorectal Polypoid Lesions: Evaluation of Contrast Enhancement in CT Colonography. European Radiology, 13, 1657-1663. http://dx.doi.org/10.1007/s00330-002-1770-y

[20] Kobayashi, K., Katsumata, T., Yoshizawa, S., Sada, M., Igarashi, M., Saigenji, K. and Otani, Y. (2005) Indications of Endoscopic Polypectomy for Rectal Carcinoid Tumors and Clinical Usefulness of Endoscopic Ultrasonography. Diseases of the Colon \& Rectum, 48, 285-291. http://dx.doi.org/10.1007/s10350-004-0765-y

[21] Yoshikane, H., Tsukamoto, Y., Niwa, Y., Goto, H., Hase, S., Mizutani, K. and Nakamura, T. (1993) Carcinoid Tumors of the Gastrointestinal Tract: Evaluation with Endoscopic Ultrasonography. Gastrointestinal Endoscopy, 39, 375-383. http://dx.doi.org/10.1016/S0016-5107(93)70109-1 